\title{
Some fall-cone tests
}

\author{
D. M. WOOD (1985). Géotechnique 35, No. 1, 64-68
}

\section{Mr M. Saffarzadeh and Mr N. K. Tovey}

Much attention has been paid to the relationship between cone penetration and the shear strength of a soil. This is for two reasons: firstly in connection with the determination of the liquid limit and secondly as a measure of shear strength itself. In the first application it is not essential that the coefficient relating cone penetration to shear strength is known, as, in theory at least, the test should be conducted under standardized conditions. However, the use of different cone angles in the Swedish and British tests raises the need for an evaluation of the coefficient even for this work, so that meaningful comparisons can be made between values obtained by the two procedures. The Author's data and the determined values of the penetration coefficient are thus to be welcomed.

The Author notes that the shear strength and penetration are related by the coefficient $k(\alpha, \chi)$
(Author's equation (1)) and while acknowledging the possible importance of adhesion etc. (as contained in the parameter $\chi$ ) he chooses to disregard it for the present study and concentrates primarily on the variation in $k_{\alpha}$ with cone angle. The choice of cone angle is fixed in both the Swedish and the British tests, but for routine approximate estimations of the shear strength from core samples etc. it is desirable to have a variety of cone angles from which to choose. Generally for stiffer soils small cone angles would be chosen. However, such small cone angles are less reliable in providing consistent results than larger cone angles because of wear of the point of the cone. Hansbo (1957) has written extensively on this point. Thus it is even more useful to have data of the kind presented by the Author. He notes that current theories are deficient and give predictions which differ from his experimental values by up to a factor of 2 . The Writers have noted for some

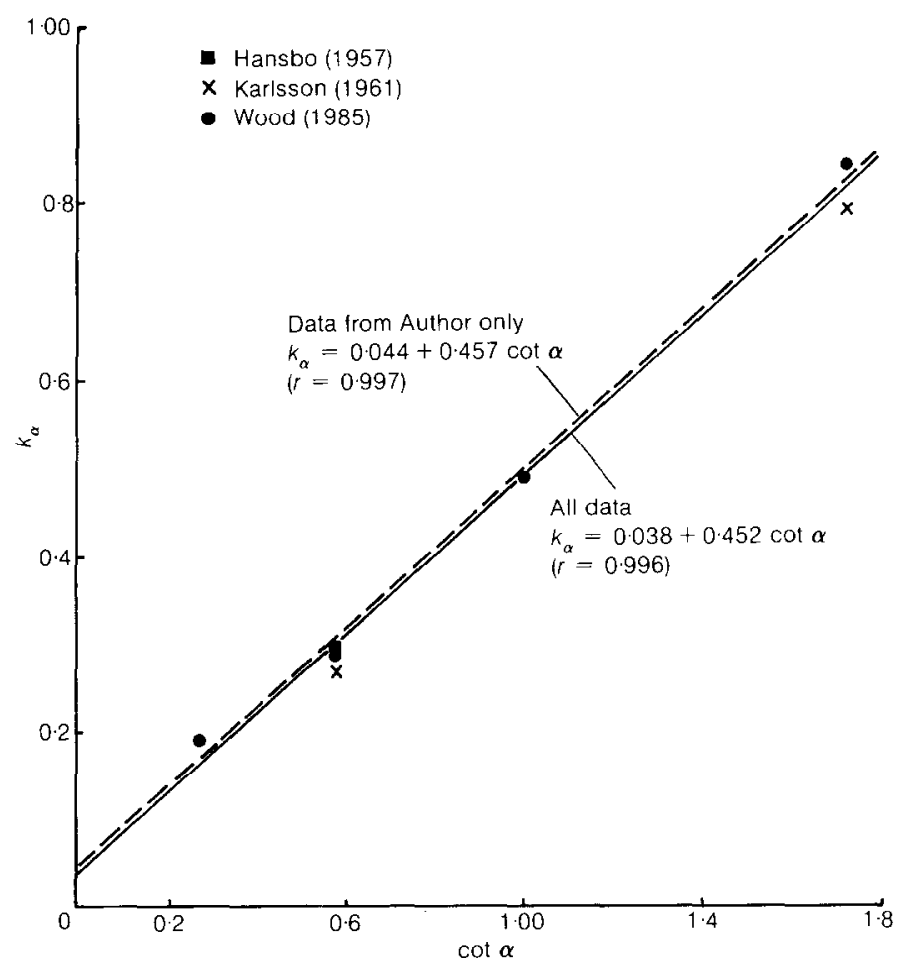

Fig. 1. Variation in penetration coefficient $k_{\alpha}$ with cone angle $\alpha$ 
Table 1. Values of penetration coefficient $k_{\alpha}$ for various cone angles $\alpha$

\begin{tabular}{c|c|c|c|c|c}
\hline $\begin{array}{c}\alpha: \\
\mathrm{deg}\end{array}$ & $\cot \alpha$ & Hansbo (1957) & Karlsson (1961) & Wood (1985) & $\begin{array}{c}\text { Values from } \\
\text { equation (2) }\end{array}$ \\
\hline 30 & 1.732 & - & 0.80 & 0.85 & 0.821 \\
45 & 1.000 & - & - & 0.49 & 0.490 \\
60 & 0.577 & 0.30 & 0.27 & 0.29 & 0.299 \\
75 & 0.268 & - & - & 0.19 & 0.160 \\
\hline
\end{tabular}

time that a close empirical relationship exists between the values of $k_{\alpha}$ and the cotangent of the whole cone angle. This contrasts with the square of the cotangent of the semicone angle in the equations (7) and (8) in the Paper. Plotting the Author's data as $k_{\alpha}$ against $\alpha$ (Fig. 1) emphasizes this relationship while a linear regression of the transformed data yields a high coefficient of correlation of 0.997 , the cquation of the line bcing

$$
k_{\alpha}=0.044+0.457 \cot \alpha
$$

This is shown as the broken line in Fig. 1.

If the data from Karlsson (1961) $\left(k_{30}=0.80\right.$; $\left.k_{60}=0 \cdot 27\right)$ and Hansbo (1957)- $\left(k_{60}=0 \cdot 30\right)$ are added then a high correlation of 0.996 is still obtained with a slight modification of the equation to

$$
k_{\alpha}=0.038+0.452 \cot \alpha
$$

These additional points are also shown in Fig. 1 together with equation (2) shown as a full line. The complete set of data together with values predicted by equation (2) are presented in Table 1.

Although correlation does not imply cause, nevertheless in this case the high value of the correlation coefficient does form the basis of a useful empirical relationship for practical use. It may also assist in developing a new theory to account for the variation in $k_{x}$ with cone angle.

\section{REFERENCES}

Hansbo, S. (1957). A new approach to the determination of the shear strength of clay by the fall-cone test. Proc. R. Swed. Geotech. Inst., No. 14.

Karlsson, R. (1961). Suggested improvements in the liquid limit test, with reference to flow properties of remoulded clays. Proc. 5th Int. Conf. Soil Mech. Fdn Engng, Paris 1, 171-184.

\section{Author's reply}

The data are somewhat sparse so the accuracy of the linear relationship that the Writers propose between cone factor $k_{\alpha}$ and $\cot \alpha$ should perhaps be regarded with some caution-as plotted in their Fig. 1 the Author's data would seem better to fit a curved (concave upwards) rather than a linear relationship. The linear relationship that the writers produce certainly cannot be extrapolated to higher cone angles: a zero cone factor is predicted for a cone angle of about $95^{\circ}$. This is not in accord with experimental expectation.

A zero cone factor would be expected for $\alpha=180^{\circ}$, a completely flat cone, for which resistance is obtained, without penetration, and for which the area of contact increases indefinitely for infinitesimal penetration. The use of a bearing capacity factor $N_{\mathrm{c}}$ avoids this problem by looking at the average pressure exerted at the soil surface and treats the cone as a finite object, not one of indefinite extent. Then the bearing capacity factor for the flat punch is seen to act as a limit towards which the values obtained from the fall-cone tests are heading (Fig. 2-average values only plotted)-whereas $k_{\alpha}$ in the expression of Saffarzadeh and Tovey has by this stage disappeared to minus infinity.

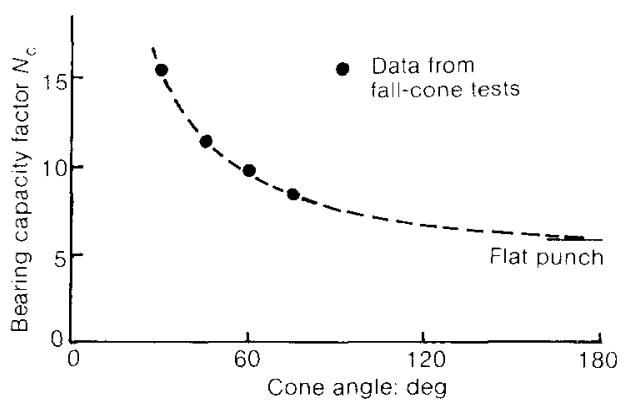

Fig. 2 